\title{
DETERMINACIÓN DEL COMPORTAMIENTO TÉRMICO DE UN INVERNADERO ESPACIAL COLOMBIANO MEDIANTE DINAMICA DE FLUIDOS COMPUTACIONAL
}

\section{DETERMINATION OF THE THERMAL BEHAVIOR OF A COLOMBIAN SPATIAL GREENHOUSE THROUGH COMPUTATIONAL FLUID DYNAMICS}

\author{
Edwin Andrés Villagran Munar ${ }^{1}$, Carlos Ricardo Bojacá Aldana ${ }^{2}$, Niccole Alejandra Rojas Bahamon ${ }^{3}$
}

\begin{abstract}
${ }^{1}$ Ing. Agrícola. M.Sc., Estudiante de Doctorado en Ciencias Ambientales y Sostenibilidad. Profesor Asistente, Facultad de Ingeniería. Corporación Universitaria Minuto de Dios, Sede Cundinamarca-Zipaquirá, Colombia, Av. 15 No 1-22 Sur Barrio La Fragüita, e-mail: evillagranm@uniminuto.edu.co, https://orcid.org/0000-0003-1860-5932; 'ng. Agrónomo Ph.D., Profesor Titular, Departamento de Ciencias Básicas y Modelado, Facultad de Ciencias Naturales e Ingeniería. Universidad Jorge Tadeo Lozano, Bogotá, Colombia, Carrera 4 No. 22-61, Módulo 6 Oficina 502, e-mail: carlos.bojaca@utadeo.edu.co, https:// orcid.org/0000-0003-0230-326X; ${ }^{3}$ Estudiante Ing. Agroecológica. Facultad de Ingeniería. Corporación Universitaria Minuto de Dios, Sede Cundinamarca-Zipaquirá, Colombia, Av. 15 No1-22 Sur Barrio La Fragüita Colombia, e-mail: nrojasbaham@ uniminuto.edu.co; https://orcid.org/0000-0002-7996-4966.
\end{abstract}

Rev.U.D.C.A Act. \& Div. Cient. 21(2):415-426, Julio-Dicembre, 2018 https://doi.org/10.31910/rudca.v21.n2.2018.1070

Artículo de acceso abierto publicado por Revista U.D.C.A Actualidad \& Divulgación Científica bajo una licencia Creative Commons CC BY-NC 4.0

\section{RESUMEN}

En Colombia, la producción de flores se lleva a cabo en invernaderos de diferentes tipos y formas geométricas, pero con la una característica común de usar ventilación natural, para control de clima. En la actualidad, el conocimiento sobre el desempeño climático de estas estructuras es escaso. El objetivo del trabajo consistió en evaluar el comportamiento térmico de un invernadero espacial en condiciones de clima diurno y nocturno. La evaluación realizada, mediante modelado computacional, empleó la dinámica de fluidos computacional (CFD, en idioma inglés), aplicada a un invernadero dedicado a la producción de clavel (Dianthus caryophyllus), bajo las condiciones meteorológicas de la Sabana de Bogotá (Colombia). Este enfoque metodológico permitió obtener los patrones de distribución térmica en el interior del invernadero, encontrando que, para las condiciones meteorológicas evaluadas, el invernadero genera unas condiciones térmicas inadecuadas para el desarrollo del cultivo. La validación del modelo CFD, se realizó comparando los resultados de las simulaciones y las temperaturas registradas en el prototipo real del invernadero, obteniendo un grado de ajuste adecuado entre los valores simulados y medidos.
Palabras clave: temperatura, simulación por ordenador, inversión térmica, Dianthus, meteorología. Tomadas de MeSH on Demand.

\section{ABSTRACT}

In Colombia, flower production takes place in greenhouses of different types and geometric shapes, but with the common feature of using natural ventilation for climate control. At present the knowledge on the climatic performance of these structures is scarce. The aim of this work was to evaluate the thermal behavior of a greenhouse under day and night climate conditions. The evaluation made by computational modeling used the computational fluid dynamics (CFD) approach applied to a greenhouse dedicated to the production of carnation (Dianthus caryophyllus) and exposed to the weather conditions of the Sabana de Bogotá (Colombia). This methodological approach allowed us to obtain the thermal distribution patterns inside the greenhouse, finding that for the meteorological conditions evaluated, the greenhouse generates inadequate thermal conditions for the development of the crop. The validation of the CFD model was carried out by comparing the results of the simulations and the temperatures recorded in the real prototype of the 
greenhouse, obtaining an adequate degree of adjustment between the simulated and measured values.

Keywords: temperature, computer simulation, thermal inversion, Dianthus, meteorology.

\section{INTRODUCCIÓN}

En Colombia, la producción de flores de corte bajo cubierta ocupa un área aproximada de 7.300ha. En esta área existe una diversidad de estructuras de invernaderos, que varían en tamaño y en forma geométrica. Dentro de los tipos más comunes, se pueden encontrar los denominados invernaderos estructurales, como el tradicional de madera, el tradicional metal-madera, el flexon, el colgante, el millenium y el tándem, los cuales, ocupan un área aproximada al 95\% del área total (Acuña et al. 2004). El 5\% restante es ocupada por el invernadero denominado tipo espacial.

En estos invernaderos, se generan condiciones micro climáticas, influenciadas por las características específicas de cada estructura. Este microclima es el conjunto de parámetros climáticos generados alrededor del dosel de las plantas, como resultado de procesos físicos, que incluyen transferencia de calor por radiación, conducción y convección y balances de masa, debido a flujos de vapor de agua y concentración de $\mathrm{CO}_{2}$. Este microclima es un factor determinante, que puede maximizar o limitar la calidad y la cantidad de la producción vegetal (van Henten et al. 2006).

La temperatura es uno de los factores más relevantes a la hora de programar las actividades de control de clima (Campen \& Bot, 2001), principalmente, porque cuando alcanza valores extremos en invernaderos pasivos (ventilados naturalmente), se limita la producción de forma parcial o total (Kittas et al. 2005) as well as their interaction, affecting the air temperature in a fan-ventilated greenhouse. On this purpose, measurements concerning both the greenhouse ventilation rate and climate variables inside and outside the greenhouse (air temperature and solar radiation inside and outside the greenhouse and outside wind speed and direction. El comportamiento térmico del volumen de aire dentro de una estructura de invernadero fluctúa en función de la cantidad de radiación solar incidente, la hermeticidad e inercia térmica, pero, primariamente, de la magnitud y la eficiencia de las tasas de ventilación natural (Coelho et al. 2006).

El control de la temperatura en los invernaderos pasivos colombianos es relevante en días calurosos de alta radiación, en los que se hace necesario evacuar los excesos térmicos producidos al interior. Bajo estas condiciones es posible alcanzar temperaturas cercanas a $45^{\circ} \mathrm{C}$ (Bojacá et al. 2009), que ocasionan desórdenes fisiológicos en las plantas (Sato et al. 2001). Adicionalmente, estos periodos de alta radiación suelen generar condiciones nocturnas secas y despejadas, que favorecen la aparición del fenómeno de inversión térmica, fenómeno, en el cual, la temperatura interior del invernadero es inferior a la del aire exterior circundante (EspinalMontes et al. 2015).

El estudio del comportamiento térmico de un invernadero, se puede realizar a través de modelos empíricos de balance de energía, experimentación en campo, termografía digital o mediante simulación numérica, aplicando técnicas, como la dinámica de fluidos computacional (CFD, por sus siglas en idioma inglés) (Chen, 2009). Esta herramienta permite realizar el análisis cualitativo y cuantitativo del comportamiento térmico de estructuras agrícolas o pecuarias, bajo diferentes escenarios de simulación (Norton et al. 2007).

El objetivo de este trabajo consistió en desarrollar y ajustar un modelo numérico CFD 3D, con el fin de estudiar el comportamiento térmico diurno y nocturno de un invernadero espacial colombiano, bajo condiciones climáticas de la sabana de Bogotá, usado para la producción de clavel (Dianthus caryophyllus).

\section{MATERIALES Y MÉTODOS}

Sitio y descripción del invernadero. El trabajo experimental, se desarrolló en un invernadero comercial con cubierta en polietileno de $8.820 \mathrm{~m}^{2}$ de superficie de suelo cubierto, perteneciente a una finca productora de clavel $\left(4^{\circ} 35^{\prime} 32.44^{\prime \prime} \mathrm{N}\right.$, 74¹2'35.14”'W, 2.510m.s.n.m.), ubicada en el municipio de Soacha (Cundinamarca, Colombia).

El invernadero evaluado estaba compuesto de 21 naves, cada una con una luz de 7,0m (Figura 1A); las alturas mínimas y máximas bajo canal fueron de 3,5 y $6 \mathrm{~m}$, respectivamente; la distancia longitudinal del invernadero fue de $60 \mathrm{~m}$ y estaba orientado en sentido norte-sur (N-S). Cada nave disponía de un área de ventilación cenital fija de 0,25m de apertura, que genera una superficie de ventilación cenital (Svc), en relación con el suelo cubierto de 3,6\%. El área total de ventilación natural, se complementaba con aperturas fijas de $2,5 \mathrm{~m}$, en los cuatro costados de la estructura, que generan una superficie de ventilación lateral (Svl), en relación con el suelo cubierto de $11,73 \%$. Todas las áreas de ventilación permanecían totalmente abiertas durante las 24 horas del día.

Se midieron las condiciones meteorológicas exteriores para las 24 horas del día, dentro del periodo comprendido entre las 00:00 horas del 07 de julio y las 23:00 horas de 05 de agosto de 2016. El registro de las variables, se realizó por medio de una estación meteorológica (Vantage Pro2 Plus, Davis Instruments, Hayward CA), que integraba sensores 

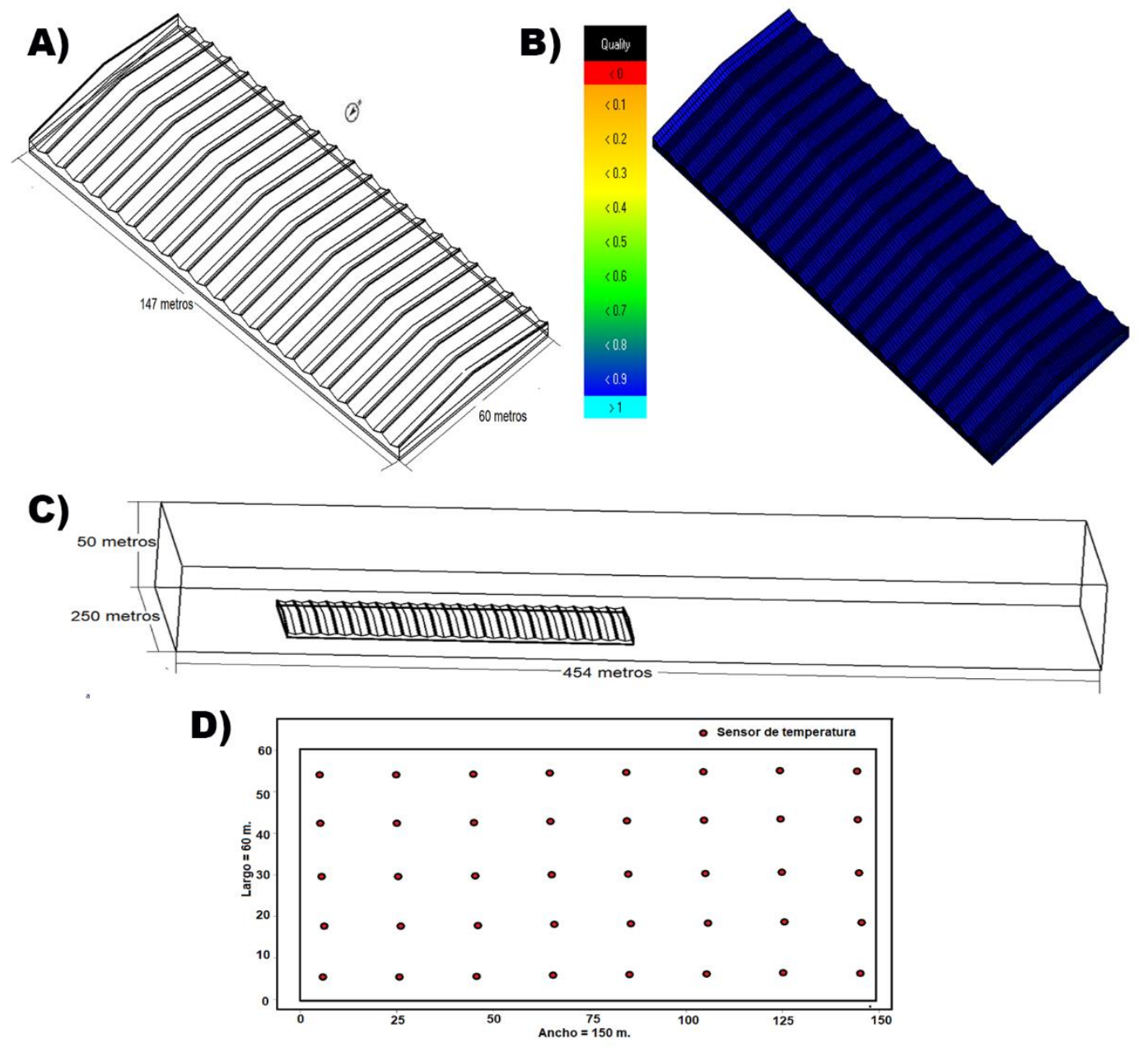

Figura 1. A) Área del invernadero espacial evaluado; B) Detalle de la calidad de la malla; C) Dominio computacional y D) Ubicación de los sensores de medición de temperatura.

de radiación global, temperatura, humedad relativa, precipitación, velocidad del viento y dirección y la frecuencia de registro fue cada 10 minutos.

Modelación y Simulación numérica. La simulación numérica por la técnica CFD permite resolver las ecuaciones gobernantes del flujo de fluidos utilizando el método de volumen finito, para convertir las ecuaciones diferenciales parciales en un conjunto de ecuaciones algebraicas (Molina-Aiz et al. 2009). Estas ecuaciones pueden ser representadas como ecuaciones de convección-difusión de un fluido para tres leyes de conservación, que incluyen las ecuaciones de momento, energía y transporte de un fluido compresible y en un campo tridimensional (3D) y se expresan así:

$$
\frac{\partial \rho \phi}{\partial t}+\nabla(\rho \phi \vec{v})=\nabla(\Gamma \nabla \phi)+\mathrm{S}
$$

(Ecuación 1)
Donde, $\rho$ es la densidad del fluido $\left(\mathrm{kg} \mathrm{m}^{-3}\right)$, es el operador nabla, $\phi$ representa la variable de concentración, es el vector de velocidad $\left(\mathrm{m} \mathrm{s}^{-1}\right), \Gamma$ es el coeficiente de difusión $\left(\mathrm{m}^{2} \mathrm{~s}^{-1}\right)$ y $S$ representa el término fuente (Piscia et al. 2012). La ecuación 1 representa la ecuación de continuidad, cuando $\phi$ es 1 ; la ecuación de energía, cuando $\phi$ es $\mathrm{T}\left({ }^{\circ} \mathrm{C}\right)$ y la ecuación de momento, cuando $\phi$ es u, v, w las velocidades en las direcciones $\mathrm{x}, \mathrm{y}, \mathrm{z}$ en $\mathrm{m} \mathrm{s}^{-1}$.

Estos conjuntos de ecuaciones fueron resueltos mediante la utilización del software comercial ANSYS Fluent (v. 17.0). La naturaleza turbulenta del flujo de aire se simuló utilizando el modelo de turbulencia estándar $k-\varepsilon$, el cual, se basa en dos ecuaciones principales: una, para $k$, que representa la energía cinética y, otra, para $\varepsilon$, que representa la tasa de disipación en tiempo y en volumen unitarios. Las ecuaciones de transporte para $k$ y $\varepsilon$ pueden ser modeladas como: 


$$
\frac{\partial}{\partial x}(\rho k)=\frac{\partial}{\partial x_{j}}\left[\left(\mu+\frac{\partial k}{x_{j}}\right) \frac{\partial k}{\partial x_{j}}\right]+G_{k}+G_{b}-\rho \epsilon-Y_{M}
$$

(Ecuación 2)

$$
\frac{\partial}{\partial t}(\rho \varepsilon)=\frac{\partial}{\partial x_{i}}\left[\left(\mu+\frac{\mu_{t}}{\sigma}\right) \frac{\partial \epsilon}{\partial x_{i}}\right]+\rho C_{1} S_{\epsilon}-\rho C_{2} \frac{\epsilon^{2}}{k+\sqrt{v \epsilon}}+C_{1 \epsilon} \frac{\epsilon}{k} C_{3 \epsilon} G_{b} k
$$

(Ecuación 3)

Donde, es viscosidad; es viscosidad turbulenta; son los números turbulentos de Prandtl, para $k$ y ; $G_{k}$ es la generación de energía cinética turbulenta, debida a los gradientes de velocidad; $G_{b}$ es generación de energía cinética turbulenta, debida a la flotabilidad; $Y_{M}$ es la dilatación fluctuante en la turbulencia, debida a la tasa de disipación global y es el coeficiente de viscosidad cinemá tica; son constantes con valores predeterminados. Este modelo estándar $k-\varepsilon$ ha sido ampliamente usado y validado en estudios enfocados a invernaderos, demostrando una precisión adecuada (Fatnassi et al. 2006; Katsoulas et al. 2006).

Todas las simulaciones consideraron la ecuación de energía, lo cual, permitió analizar el campo escalar de temperaturas al interior del invernadero; asimismo, el modelado del fenómeno de flotabilidad, que impulsa el movimiento del aire bajo condición de velocidad exterior bajo, se realizó por medio de la aproximación de Boussinesq, que se describe de la siguiente forma:

$$
\rho=\rho_{\text {ref }}\left[1-\beta\left(T-T_{\text {ref }}\right)\right]
$$

(Ecuación 4)

Donde, $\rho_{\text {ref }}$ es la densidad constante del flujo; $T_{\text {ref }}$ es la temperatura real y $\beta$ es el coeficiente de expansión térmica $(\beta$ $=0,00329$ para el aire). La aproximación de Boussinesq es válida si las diferencias de temperaturas que aparecen en el dominio computacional no son demasiado grandes $\beta\left(T-T_{\text {ref }}\right)$ $<1$, situación que, generalmente, ocurre en el estudio de microclima de invernaderos (Baeza et al. 2006).

El modelo de radiación seleccionado fue el de ordenadas discretas (DO), con discretización angular. Este modelo permite realizar análisis de clima en condiciones de periodo nocturno, simulando y resolviendo el fenómeno de radiación, desde el suelo del invernadero hacia el ambiente exterior (Iglesias et al. 2009).

El método seleccionado de solución de flujo del fluido fue el de volúmenes finitos, en el que el resultado se obtiene mediante la discretización e integración de las ecuaciones de transporte, a través de la división del dominio computacional, en una malla no estructurada.
Dominio computacional y generación de la malla. El software de preprocesamiento ANSYS ICEM CFD (v. 17,0), se utilizó para generar un dominio computacional, compuesto por el invernadero y sus alrededores, con el fin de garantizar la no afectación de la solución numérica del campo de flujo fuera del invernadero y para permitir una definición apropiada de la capa límite atmosférica (Rico-Garcia et al. 2011). Las dimensiones del dominio computacional fueron de 454, 260 y 50m, para los ejes x, y, z, respectivamente (Figura 1C); este tamaño, se determinó siguiendo las pautas para el cálculo de CFD del entorno eólico alrededor de los edificios (Tominaga et al. 2008)guidelines are required that summarize important points in using the CFD technique for this purpose. This paper describes guidelines proposed by the Working Group of the Architectural Institute of Japan (AIJ. El dominio computacional estuvo compuesto por una malla no estructurada de elementos cúbicos, para los volúmenes 3D y elementos cuadrados, para las superficies 2D. La malla, se dividió en 46,980,432 volúmenes discretizados en el espacio, número de elementos que se obtuvo luego de verificar la independencia de las soluciones numéricas del flujo de aire a tamaños de malla con un número superior e inferior de elementos, de acuerdo con el procedimiento reportado por He et al. (2017); cada una de estas mallas, se construyó empleando el paquete de cómputo ANSYS (v. 17.0). Un criterio fundamental para establecer la precisión de las soluciones obtenidas, mediante CFD, consiste en evaluar la calidad de la malla (Figura 1B). Los parámetros de calidad evaluados fueron el tamaño de las celdas y la variación del tamaño de celda a celda, encontrando que, un $92,9 \%$ de las celdas, estaban dentro del intervalo de calidad alta (0,95-1). También, se evaluó el ortogonal quality, donde el valor mínimo obtenido fue de 0,91 , resultados que se clasifican dentro del rango de alta calidad (Flores-Velázquez et al. 2015).

El software comercial CFD ANSYS FLUENT (v. 17,0), se utilizó para realizar dos conjuntos de simulaciones, unas de clima diurno (6-17 horas) y, otras, de clima nocturno (0-5 y 1823 horas). Los criterios de convergencia del modelo fueron establecidos en $10^{-8}$, para la ecuación de energía y en $10^{-6}$, para las ecuaciones de continuidad, momento y turbulencia (Baxevanou et al. 2017).

Condiciones de frontera. El límite superior del dominio y las superficies paralelas al flujo fueron fijadas con condiciones de frontera de propiedades simétricas, para no generar pérdidas de fricción del flujo de aire en contacto con estas superficies. Las simulaciones consideraron las características atmosféricas del municipio de Soacha, tales como presión atmosférica de 74,993Pa, viscosidad del aire igual a 1,7E$05 \mathrm{~kg} \mathrm{~m}^{-1} \mathrm{~s}^{-1}$ y gravedad de $9,8 \mathrm{~ms}^{-2}$. Al límite inferior y las paredes del invernadero, se les fijó una condición de frontera de pared antideslizante. Otras propiedades del polietileno y del suelo agrícola ,como calor específico $(C p)$, conductividad 
térmica $(k)$ y densidad $(\rho)$, fueron establecidas, de acuerdo con Villagrán et al. (2012). Los límites perimetrales del dominio computacional, se establecieron como límites de entrada de aire o salida de presión, según el caso a evaluar. Se consideró un perfil uniforme de velocidad del viento evaluando velocidades con valores entre 0,32 y $2,61 \mathrm{~ms}^{-1}$ y valores de temperatura medios, para cada hora del día. Dichos valores fueron establecidos a partir de la información climática recopilada en el periodo de medición experimental.

Otros parámetros de entrada que son necesarios para alimentar el modelo de radiación son las propiedades ópticas del material de cubierta, establecidas para el fenómeno de radiación infrarroja de onda larga. Los valores para el poli- etileno transparente fueron coeficiente absorción $(\alpha)$, de 0,69; coeficiente de transmisión $(T)$, de 0,19 y coeficiente de reflexión $(\rho)$, de 0,11 . El modelo no incluyó cultivo alguno y, adicionalmente, se asumió una hermeticidad máxima del invernadero. Estas simplificaciones son válidas puesto que son aplicadas a cada una de los casos simulados y los errores que se puedan derivar tendrán el mismo grado de magnitud, para cada escenario.

Escenarios considerados. La evaluación del comportamiento térmico, se realizó asumiendo las condiciones meteorológicas medias para cada hora evaluada (Tabla 1) y la configuración de ventilación estándar del invernadero estudiado que, para este caso, fue ventilación lateral combinada con ventilación de techo, para las 24 horas del día.

Tabla 1. Condiciones meteorológicas medias, usadas como parámetros de entrada al modelo CFD-3D.

\begin{tabular}{|c|c|c|c|c|c|}
\hline Hora & $\begin{array}{c}\text { Temperatura } \\
\text { media exterior }\left({ }^{\circ} \mathrm{C}\right)\end{array}$ & $\begin{array}{l}\text { Temperatura } \\
\text { del cielo }\left({ }^{\circ} \mathrm{C}\right)\end{array}$ & $\begin{array}{c}\text { Vel_Viento } \\
\left(\mathrm{ms}^{-1}\right)\end{array}$ & $\begin{array}{c}\text { Dir_Viento } \\
\left(_{\left({ }^{\circ}\right)}\right.\end{array}$ & $\begin{array}{l}\text { Radiación Solar } \\
\left(w^{-2}\right)\end{array}$ \\
\hline 0 & 12,45 & 0 & 0,35 & 191,73 & 0 \\
\hline 1 & 12,25 & 0 & 0,33 & 175,14 & 0 \\
\hline 2 & 11,82 & 0 & 0,33 & 166,86 & 0 \\
\hline 3 & 11,57 & 0 & 0,32 & 159,61 & 0 \\
\hline 4 & 11,31 & 0 & 0,35 & 166,32 & 0 \\
\hline 5 & 11,03 & 0 & 0,32 & 204,25 & 0 \\
\hline 6 & 10,90 & NA & 0,35 & 136,55 & 0,97 \\
\hline 7 & 11,24 & NA & 0,52 & 135,68 & 43,29 \\
\hline 8 & 12,92 & NA & 0,75 & 131,72 & 147,29 \\
\hline 9 & 15,11 & $\mathrm{NA}$ & 1,68 & 155,52 & 333,09 \\
\hline 10 & 16,50 & NA & 1,7 & 176,64 & 502,28 \\
\hline 11 & 17,45 & NA & 1,69 & 174,58 & 628,57 \\
\hline 12 & 17,71 & NA & 2,05 & 175,71 & 633,97 \\
\hline 13 & 17,95 & NA & 2,56 & 170,32 & 419,12 \\
\hline 14 & 18,32 & NA & 2,61 & 176,61 & 501,91 \\
\hline 15 & 17,98 & NA & 2,55 & 194,66 & 399,43 \\
\hline 16 & 17,39 & NA & 2,38 & 198,64 & 290,87 \\
\hline 17 & 16,37 & NA & 2,11 & 207,94 & 164,72 \\
\hline 18 & 15,29 & 0 & 1,44 & 210,33 & 44,08 \\
\hline 19 & 14,24 & 0 & 0,72 & 197,49 & 0,22 \\
\hline 20 & 13,61 & 0 & 0,52 & 195,81 & 0 \\
\hline 21 & 13,37 & 0 & 0,50 & 173,19 & 0 \\
\hline 22 & 13,16 & 0 & 0,46 & 171,01 & 0 \\
\hline 23 & 12,75 & 0 & 0,40 & 198,74 & 0 \\
\hline
\end{tabular}

NA: no aplica para el escenario evaluado. 
Evaluación del modelo desarrollado. La validación del modelo CFD, se efectuó, a través de la comparación entre los resultados de las simulaciones del comportamiento térmico del invernadero, para cada hora evaluada y el registro experimental, para la misma frecuencia temporal de la temperatura dentro del invernadero real, durante el periodo de evaluación. La temperatura del aire, se registró por medio de treinta termopares tipo $\mathrm{T}$ (cobre-constantan), conectados a un número igual de registradores de datos (Cox-Tracer Junior, Escort DLS, Edison, NJ). Los conjuntos de termopares, se desplegaron uniformemente a lo largo del eje transversal y longitudinal del invernadero. Los termopares, se colocaron dentro de cilindros de PVC blanco, para reflejar la luz solar directa, a una altura de $1,5 \mathrm{~m}$ de la superficie del suelo. Las estaciones en el interior del invernadero registraron datos cada 10 minutos.

\section{RESULTADOS Y DISCUSIÓN}

Evaluación del modelo. La temperatura media del aire interno fue obtenida, a través de datos puntuales de cada una de las simulaciones horarias en estado estacionario, donde cada valor extraído coincidía con la ubicación de los sensores del registro experimental (Figura 1D). En la figura 2, se puede observar cómo las curvas de temperatura diaria simulada presentan una tendencia similar a la curva de temperatura media medida. Cuantitativamente, al comparar los datos medidos y simulados, se obtuvo un error medio absoluto (MAE), de $0,48^{\circ} \mathrm{C}$, un error porcentual absoluto medio
(MAPE), de 3,37\% y el error cuadrático medio (MSE), de $0,35^{\circ} \mathrm{C}$, valores que permiten garantizar que, el uso del modelo CFD, es adecuado para la evaluación y la descripción del comportamiento térmico del invernadero.

Periodo diurno. Las simulaciones numéricas permitieron calcular la evolución horaria del valor de la temperatura media interior del invernadero, para las condiciones de clima diurno (6 a 17 horas). El comportamiento de la variable osciló entre un valor mínimo de $11,17^{\circ} \mathrm{C}$, para la hora 6 y un valor máximo de $23,98^{\circ} \mathrm{C}$, para la hora 13 (Tabla 2). Lo anterior genera unos diferenciales térmicos entre el interior y el exterior del invernadero $\left(\Delta \mathrm{T}=\mathrm{T}_{\text {media interior }} \mathrm{T}_{\text {media exterior }}\right)$, de $0,27 \mathrm{y}$ $6,03^{\circ} \mathrm{C}$, respectivamente. Estos valores de $\Delta \mathrm{T}$, se encuentran fuertemente influenciados por el valor de la radiación solar presentados para cada hora evaluada y por la ganancia térmica que se va generando en el volumen de aire interior, debido el efecto invernadero, producido por el material de cubierta.

La temperatura interior del invernadero, para las horas 6 y 7 , se encuentra por debajo del valor mínimo requerido para el cultivo de clavel (Dianthus caryophyllus), que debe ser de $15^{\circ} \mathrm{C}$ (Newman, 1999), lo que puede llegar a producir flores aplanadas con apertura parcial. Por otro lado, entre las horas 11 y 15, la temperatura se encontró por encima del valor máximo requerido de $22^{\circ} \mathrm{C}$ (Newman, 1999). Estos valores elevados de temperatura generan un ciclo vegetativo más acelerado con una consecuente pérdida de calidad en el producto

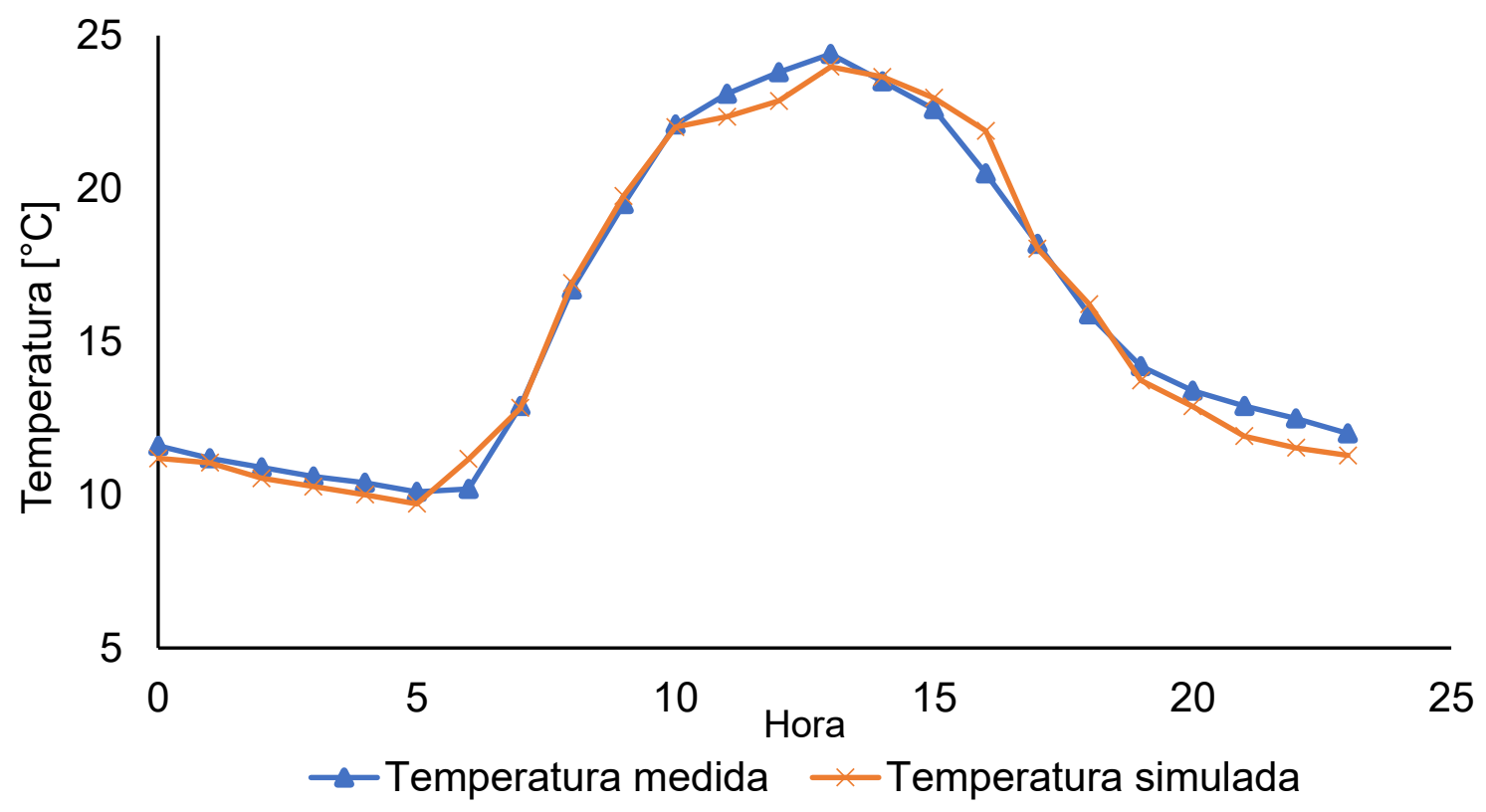

Figura 2. Perfil de temperatura horario medido y simulado dentro del invernadero espacial. 
Tabla 2. Temperatura media interior y diferencia térmica entre el interior y el exterior del invernadero, para los escenarios simulados.

\begin{tabular}{|c|c|c|c|c|c|}
\hline \multicolumn{3}{|c|}{ Periodo nocturno } & \multicolumn{2}{c|}{ Periodo Diurno } \\
\hline Hora & $\begin{array}{c}\text { Temperatura media } \\
\text { interior }\left({ }^{\circ} \mathbf{C}\right)\end{array}$ & $\begin{array}{c}\text { Diferencial térmico } \\
\left(\Delta \mathrm{T},{ }^{\circ} \mathbf{C}\right)\end{array}$ & Hora & $\begin{array}{c}\text { Temperatura } \\
\text { media interior }\left({ }^{\circ} \mathrm{C}\right)\end{array}$ & $\begin{array}{c}\text { Diferencial térmico } \\
\left(\Delta \mathrm{T},{ }^{\circ} \mathrm{C}\right)\end{array}$ \\
\hline 0 & 11,19 & $-1,26$ & 6 & 11,17 & 0,27 \\
\hline 1 & 11,05 & $-1,2$ & 7 & 12,84 & 3,6 \\
\hline 2 & 10,54 & $-1,28$ & 8 & 16,91 & 4,99 \\
\hline 3 & 10,27 & $-1,3$ & 9 & 19,76 & 5,51 \\
\hline 4 & 10,01 & $-1,3$ & 10 & 22,01 & 4,9 \\
\hline 5 & 9,71 & $-1,32$ & 11 & 22,35 & 5,15 \\
\hline 18 & 16,23 & 0,94 & 12 & 22,86 & 6,03 \\
\hline 19 & 13,74 & $-0,5$ & 13 & 23,98 & 5,33 \\
\hline 20 & 12,89 & $-0,72$ & 14 & 23,65 & 4,98 \\
\hline 21 & 11,92 & $-1,45$ & 15 & 22,96 & 4,5 \\
\hline 22 & 11,54 & $-1,62$ & 16 & 21,89 & 1,67 \\
\hline 23 & 11,29 & $-1,46$ & 17 & 18,04 & \\
\hline
\end{tabular}

final, generando tallos más cortos y flores más pequeñas, con una vida útil de florero más corta (Boshell, 2009).

La figura 3 presenta una vista de planta a $1.5 \mathrm{~m}$ sobre el nivel del suelo de la distribución térmica en el interior del invernadero, donde, cualitativamente, se puede observar que, para las horas 8, 12 y 16 (Figura 3 A-C), existen algunas diferencias térmicas en el volumen de aire interno del invernadero. En la hora 8, se puede observar un comportamiento térmico homogéneo, con un valor medio de $16,91^{\circ} \mathrm{C}$ y un $\Delta \mathrm{T}$ medio, de $3,99^{\circ} \mathrm{C}$, con algunas temperaturas inferiores en $1,2^{\circ} \mathrm{C}$, cerca de las aperturas laterales y frontales del invernadero, respecto a la media obtenida (Figura 3A). Para la hora 12 , la temperatura media obtenida fue de $22,86^{\circ} \mathrm{C}$ y un $\Delta \mathrm{T}$ medio, de $5,15^{\circ} \mathrm{C}$, mientras que las temperaturas mínimas fueron $2,3^{\circ} \mathrm{C}$, menores con respecto a la temperatura media. En este caso, las temperaturas mínimas se presentaron, aproximadamente, en un $25 \%$ del volumen del invernadero, justamente, en la zona de influencia de la apertura lateral de barlovento (Figura 3B). Por último, para la hora 16, el valor medio de temperatura interior obtenido fue de $21,89^{\circ} \mathrm{C}$ y un $\Delta \mathrm{T}$ medio, de $4,5^{\circ} \mathrm{C}$. En un volumen aproximado de $40 \%$ del invernadero y en las zonas de influencia de las aperturas perimetrales del invernadero, se obtuvieron temperaturas inferiores en $1,89^{\circ} \mathrm{C}$, con respecto a la temperatura media (Figura 3C). Esto permite concluir que el invernadero genera unas condiciones térmicas heterogéneas influenciadas, posiblemente, por una baja inercia térmica de la estructura y deficiencias de ventilación de la misma. Esta heterogeneidad térmica, se traduce en producciones diferenciadas, tanto en calidad como en cantidad.
Los flujos de aire generados en el interior del invernadero presentan un comportamiento similar para las horas evaluadas, mostrando un comportamiento de velocidades altas en la ventana frontal de barlovento, con valores de 0,62, 1,58 y $2,21 \mathrm{~ms}^{-1}$, para las horas 8,12 y 16 , respectivamente. A medida que avanzan en el eje longitudinal del invernadero, se desaceleran, exhibiendo valores de 0,21, 0,53 y 0,27ms 1 , zonas que coinciden con las áreas de alta temperatura, para cada caso. La velocidad de los flujos de aire vuelve a aumentar en el área de influencia de la ventana frontal de sotavento (Figura $3 \mathrm{D}-\mathrm{F}$ ). Se puede concluir que, este tipo de invernaderos, presentan insuficiencias en la ventilación, lo cual, genera condiciones microclimaticas heterogenias asociadas a una baja relación de la superficie de ventana cenital en comparación con la superficie de ventana lateral (Svc/Svl) que, para este caso, fue de 0,306, cuando la recomendación del valor para invernaderos ventilados de forma pasiva debe estar en el intervalo de 0,5 < Svc/Svl < 1 (Kittas et al. 1997).

Periodo nocturno. En la tabla 2, se reporta el comportamiento térmico del invernadero para el periodo comprendido entre las 18 y las 5 horas. La temperatura media interior del invernadero, calculada bajo esta condición, osciló entre un valor máximo de $16,23^{\circ} \mathrm{C}$ y un valor mínimo de $9,71^{\circ} \mathrm{C}$, para las horas 18 y 5 , respectivamente.

El análisis del comportamiento horario del valor de la temperatura indicó que, para las horas de la noche, a excepción de la hora 5, el invernadero se mantiene dentro de los rangos de temperatura nocturna óptima $\left(10-13^{\circ} \mathrm{C}\right)$, recomendada para el cultivo de clavel (Dianthus caryophyllus). Aunque 

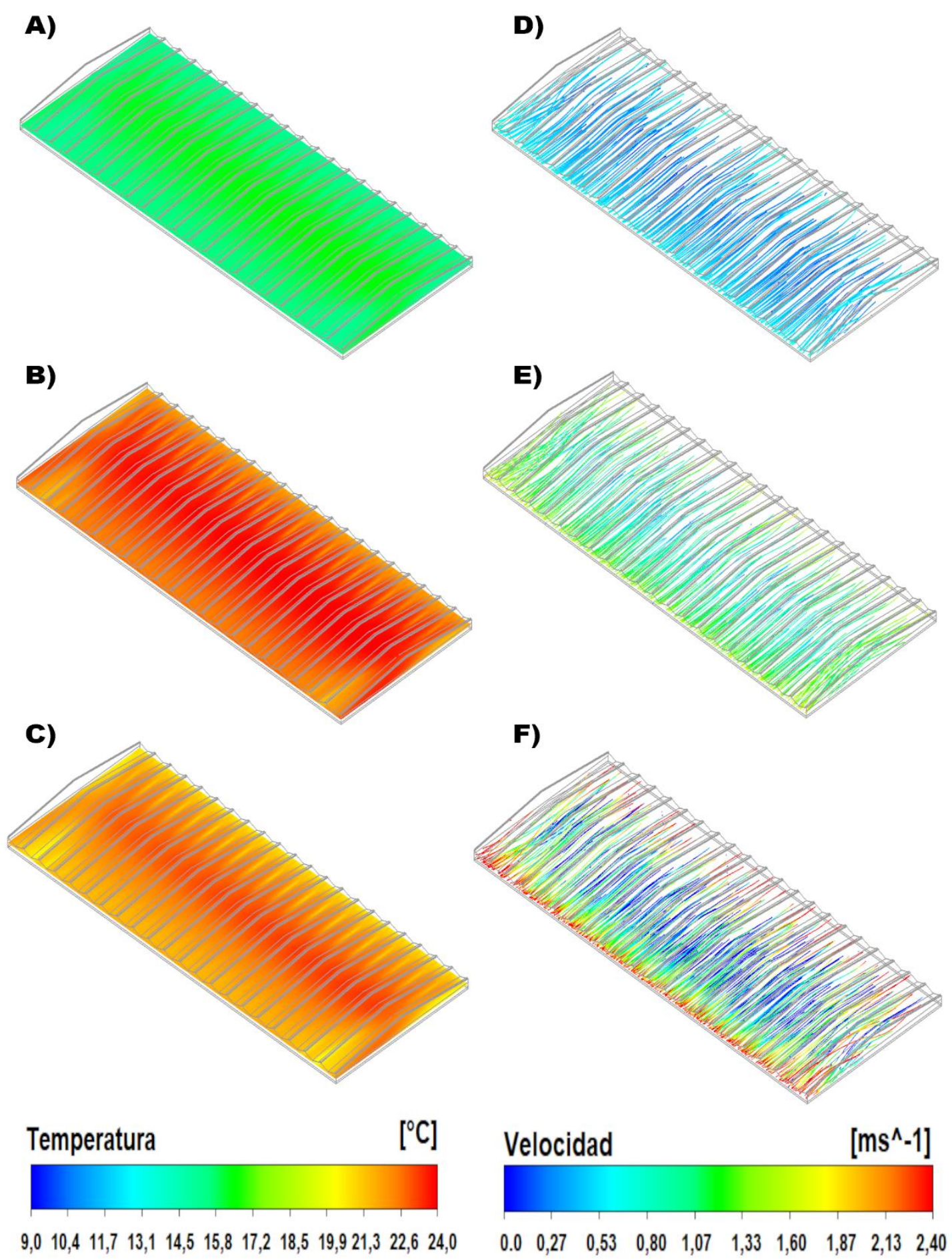

Figura 3. Contornos de temperatura $\left({ }^{\circ} \mathrm{C}\right)$, simulados para: A) hora 8; B) hora 12 y C) hora 16 y contornos de flujo de aire $\left(\mathrm{ms}^{-1}\right)$, simulados para: D) hora 8; E) hora 12 y F) hora 16. 
se debe resaltar que los valores de $\Delta \mathrm{T}$ oscilan entre $0,94 \mathrm{y}$ $-1,62^{\circ} \mathrm{C}$, con un alto porcentaje de $\Delta \mathrm{T}$, con valor negativo, lo que es un indicativo que, bajo las condiciones meteorológicas predominantes presentes en la fase de evaluación, el aire interior del invernadero, se encuentra en condiciones de inversión térmica. Este fenómeno, se genera, principalmente, por una pérdida acelerada de calor, a través de la cubierta y del flujo de calor, extraído del interior del invernadero, a través de las aperturas cenitales fijas (Teitel et al. 2008) y, en este, a través las aperturas perimetrales, permanecieron abiertas en las horas de la noche. Los flujos de aire generados por esta configuración de ventilación presentaron un movimiento desde la ventana frontal de barlovento en dirección a la ventana frontal de sotavento, con velocidades medias de $0,35,0.21$ y $0,17 \mathrm{~ms}^{-1}$, para las 20,0 y 4 horas, respectivamente (Figura $4 \mathrm{D}-\mathrm{F})$.
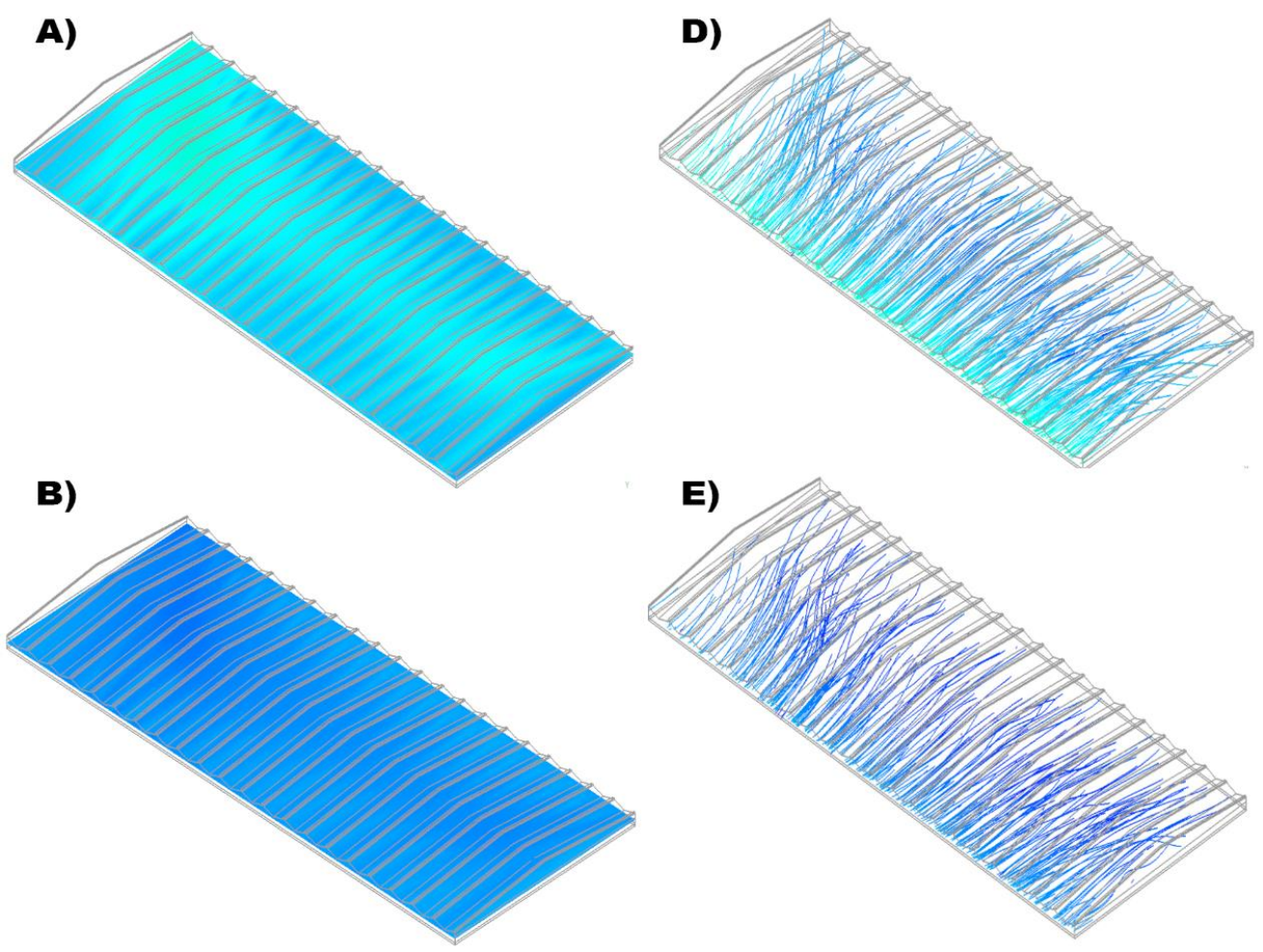

C)
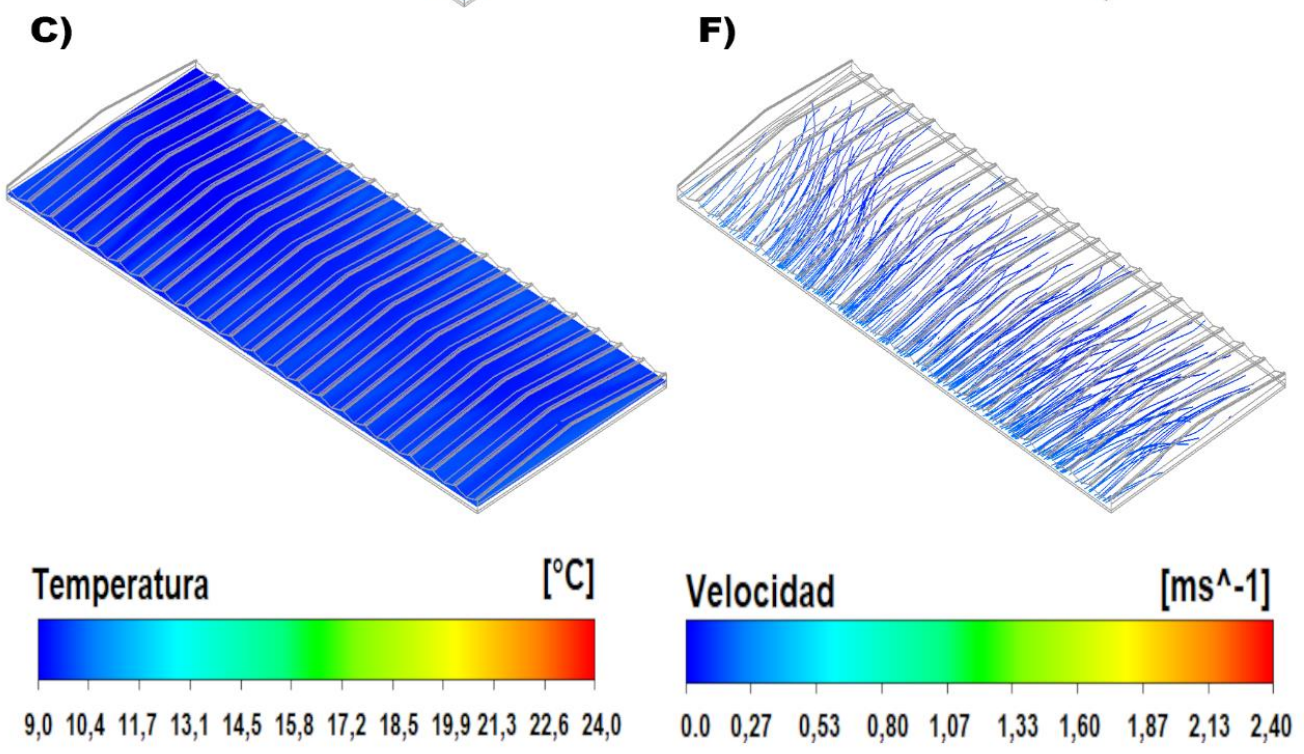

Figura 4. Contornos de temperatura $\left({ }^{\circ} \mathrm{C}\right)$, simulados para: A) hora 20; $\mathrm{B}$ ) hora 0 y C) hora 4 y contornos de flujo de aire (ms ${ }^{1}$ ), simulados para: D) hora 20; E) hora 0 y F) hora 4. 
Se debe resaltar que el periodo de evaluación no correspondió a la época donde se presenta el fenómeno de heladas o temperaturas mínimas críticas en la sabana de Bogotá. Por lo anterior, es deducible que, cuando se presenten estas condiciones, el comportamiento térmico del invernadero será aún más deficiente, ya que la pérdida energética, bajo esas condiciones, es mucho más acelerada en el tiempo; por lo tanto, las consecuencias agronómicas pueden contemplar la pérdida total de la producción (tallos y flores), por efecto del daño por frío o congelación o una pérdida parcial, cuando las temperaturas del aire interior descienden a valores inferiores a los $2^{\circ} \mathrm{C}$ (Boshell, 2009). Bajo estas condiciones, se limitará el fenómeno de fotosíntesis, debido a la interrupción de las rutas energéticas en la planta.

En la figura 4, se presenta el comportamiento térmico para las 20, 0 y 4 horas, donde el valor de la temperatura media, en una vista de planta a $1.5 \mathrm{~m}$ del suelo del invernadero, encontrando valores de $12,89,11,19$ y $10,01^{\circ} \mathrm{C}$, respectivamente. Es posible identificar cómo la estructura de invernadero no tiene la capacidad de mantener la ganancia energética almacenada durante el periodo diurno, cediéndola, de forma acelerada al exterior, generando la inversión térmica. El comportamiento de la temperatura es homogéneo en un alto porcentaje del volumen de aire interior y se logran identificar áreas con valores de temperaturas superiores a las medias registradas que, incluso, alcanzan valores similares a la temperatura exterior. Esto sucede justo en las zonas de influencia de las aperturas perimetrales y es una tendencia para las tres horas evaluadas. Se debe mencionar que una de las labores que se suele realizar para limitar o eliminar la presencia del fenómeno de inversión térmica es ventilar, a través de las aperturas laterales y cenitales fijas (Montero et al. 2013), por lo que podemos reafirmar lo anteriormente mencionado y las deficiencias o limitantes que presenta este tipo de invernadero, en términos de tasas de ventilación. El modelo evaluado permanece con las aperturas totalmente abiertas durante las 24 horas del día, por lo que la recomendación es buscar estrategias, que permitan aumentar la estanqueidad térmica del invernadero espacial.

Como conclusión, se puede mencionar que el uso de CFD, a partir de modelos 3D, permiten desarrollar una descripción cualitativa y cuantitativa del comportamiento térmico de estructuras de invernadero, bajo condiciones climáticas de la sabana de Bogotá. La evaluación experimental del modelo mostró un ajuste cualitativo y cuantitativo óptimo, con un comportamiento en tendencia similar, entre las temperaturas simuladas y medidas, en la estructura real. Se comprobó que las condiciones térmicas generadas por el invernadero tipo espacial no son las óptimas recomendadas para la producción de clavel (Dianthus caryophyllus), ya que, durante el periodo diurno, un alto porcentaje de las horas evaluadas, se encuentran por encima del máximo sugerido. Adicionalmente, es recomendable establecer estrategias de manejo climático, para prevenir o limitar al máximo la aparición del fenómeno de inversión térmica, ya que, bajo los escenarios evaluados, las condiciones micro climáticas nocturnas en el interior del invernadero muestran predominio de dicho fenómeno, situación que puede ser aún más severa, bajo condiciones de helada. El enfoque utilizado en este trabajo, se puede usar como una herramienta para la caracterización de otros tipos de invernaderos, usados para la producción hortícola o de ornamentales, bajo diferentes condiciones climáticas.

Agradecimientos: Los autores agradecen al Servicio Nacional de Aprendizaje (SENA), la Asociación Colombiana de Exportadores de Flores (Asocolflores) y al Centro de Innovación de la Floricultura Colombiana (Ceniflores). Conflicto de intereses: El manuscrito fue preparado y revisado con la participación de todos los autores, quienes declaramos que no existe conflicto. Financiación: Este estudio fue financiado el SENA, Ceniflores y Asocolflores, en el desarrollo del proyecto de investigación denominado Generación de una herramienta de diseño u optimización de ventilación natural de los invernaderos dedicados a la producción de flores de corte en cuatro subregiones de la Sabana de Bogotá, mediante el uso de herramientas de simulación basadas en la técnica Dinámica de Fluidos Computacional (CFD).

\section{REFERENCIAS}

1. ACUÑA, J.F.; BUSTAMANTE, N.; CASALLAS, L.F.; GUTIERREZ, N. 2004. Caracterización de los invernaderos para cultivo de flores en la sabana de Bogotá. En: Memorias del VI Congreso Latinoamericano para el desarrollo y aplicación de plásticos en la agricultura. CIDAPA Bogotá. p.57-64.

2. BAEZA, E.J.; PÉREZ-PARRA, J.J.; LÓPEZ, J.C.; MONTERO, J.I. 2006. CFD study of the natural ventilation performance of a parral type greenhouse with different numbers of spans and roof vent configurations. Acta Horticulturae. 719:333-338. https://doi. org/10.17660/ActaHortic.2006.719.37

3. BAXEVANOU, C.; FIDAROS, D.; BARTZANAS, T.; KITTAS, C. 2017. Yearly numerical evaluation of greenhouse cover materials. Computers and Electronics in Agriculture. 149(1):54-70. https://doi.org/10.1016/j. compag.2017.12.006

4. BOJACÁ, C.R.; GIL, R.; COOMAN, A. 2009. Use of geostatistical and crop growth modelling to assess the variability of greenhouse tomato yield caused by 
spatial temperature variations. Computers and Electronics in Agriculture. 65(2):219-227. https://doi. org/10.1016/j.compag.2008.10.001

5. BOSHELL, J. 2009. Manejo del riesgo climático en la floricultura colombiana. Ceniflores (Colombia). 97p.

6. CAMPEN, J.B.; BOT, G.P.A. 2001. SE structures and environment:design of a low-energy dehumidifying system for greenhouses. J. Agricultural Engineering Research: 78(1):65-73. https://doi.org/10.1006/ jaer.2000.0633

7. CHEN, Q. 2009. Ventilation performance prediction for buildings: A method overview and recent applications. Building and Environment. 44(4):848-858. https://doi.org/10.1016/j.buildenv.2008.05.025.

8. COELHO, M.; BAPTISTA, F.; FITAS DA CRUZ, V.; GARCIA, J.L. 2006. Comparison of four natural ventilation systems in a Mediterranean greenhouse. Acta Hort. 719:157-171. https://doi.org/10.17660/ActaHortic.2006.719.15

9. ESPINAL-MONTES, V.; LOPEZ-CRUZ, I.; ROJANOAGUILAR, A.; ROMANTCHIK-KRIUCHOVA, E.; RAMIREZ-ARIAS, A. 2015. Determinación de los gradientes térmicos nocturnos en un invernadero usando dinámica de fluidos computacional. Agrociencia. 49(3):233-247.

10. FLORES-VELÁZQUEZ, J.; VILLARREAL-GUERRERO, F. 2015. Diseño de un sistema de ventilación forzada para un invernadero cenital usando CFD. Revista Mexicana de Ciencias Agrícolas. 6(2):303-316.

11. FATNASSI, H.; BOULARD, T.; PONCET, M.C. 2006. Optimisation of greenhouse insect screening with computational fluid dynamics. Biosystems Engineering. 93(3):301-312. http://dx.doi.org/10.1016/j.biosystemseng.2005.11.014

12. HE, X.; WANG, J.; GUO, S.; ZHANG, J.; WEI, B.; SUN, J.; SHU, S. 2017. Ventilation optimization of solar greenhouse with removable back walls based on CFD. Computers and Electronics in Agriculture. 149(1):16-25. https://doi.org/10.1016/j.compag.2017.10.001

13. IGLESIAS, N.; MONTERO, J.I.; MUÑOZ, P.; ANTÓN, A. 2009. Estudio del clima nocturno y el empleo de doble cubierta de techo como alternativa pasiva para aumentar la temperatura nocturna de los inverna- deros utilizando un modelo basado en la Mecánica de Fluidos Computacional (CFD). Hort. Argentina. 28:18-23.

14. KATSOULAS, N.; BARTZANAS, T.; BOULARD, T.; MERMIER, M.; KITTAS, C. 2006. Effect of vent openings and insect screens on greenhouse ventilation. Biosystems Engineering. 93(4):427-436. https://doi. org/10.1016/j.biosystemseng.2005.01.001

15. KITTAS, C.; KARAMANIS, M.; KATSOULAS, N. 2005. Air temperature regime in a forced ventilated greenhouse with rose crop. Energy and Buildings. 37(8):807812. https://doi.org/10.1016/j.enbuild.2004.10.009

16. KITTAS, C.; BOULARD, T.; PAPADAKIS, G. 1997. Natural ventilation of a greenhouse with ridge and side openings: sensitivity to temperature and wind effects. Trans. ASAE. 40(2):415-425. https://doi. org/10.13031/2013.21268

17. MOLINA-AIZ, F.D.; VALERA, D.L.; PEÑA, A.A.; GIL, J.A.; LÓPEZ, A. 2009. A study of natural ventilation in an Almería-type greenhouse with insect screens by means of tri-sonic anemometry. Biosystems Engineering. 104(2):224-242. https://doi.org/10.1016/j. biosystemseng.2009.06.013

18. MONTERO, J.I.; MUÑOZ, P.; SÁNCHEZ-GUERRERO, M.C.; MEDRANO, E.; PISCIA, D.; LORENZO, P. 2013. Shading screens for the improvement of the night-time climate of unheated greenhouses. Span. J. Agric. Res. 11:32-46. http://dx.doi.org/10.5424/ sjar/2013111-411-11

19. NEWMAN, S. 1999. Efectos del clima en la fisiología de la floración. En: Lee, R. (ed). Clima, fisiología y producción de cultivos bajo invernadero. Cuadernos del Centro de Investigaciones y Asesorías Agroindustriales - CIAA. Fundación Universidad de Bogotá Jorge Tadeo Lozano, Bogotá, p.27-34.

20. NORTON, T.; SUN, D.-W.; GRANT, J.; FALLON, R.; DODD, V. 2007. Applications of computational fluid dynamics (CFD) in the modelling and design of ventilation systems in the agricultural industry: A review. Bioresource Technology. 98(12):2386-2414. https:// doi.org/10.1016/j.biortech.2006.11.025

21. PISCIA, D.; MONTERO, J.I.; BAEZA, E.; BAILEY, B.J. 2012. A CFD greenhouse night-time condensation model. Biosystems Engineering. 111(2):141-154. https://doi.org/10.1016/j.biosystemseng.2011.11.006 
22. RICO-GARCÍA, E.; SOTO-ZARAZÚA, G.M.; ALATORRE-JACOME, G.A.; De la Torre-Gea, O. 2011. Aerodynamic study of greenhouses using computational fluid dynamics. Internal J. Physical Sciences. 6(28):6541-6547. https://doi.org/10.5897/ IJPS11.852

23. SATO, S.; PEET, M.M.; GARDNER, R.G. 2001. Formation of parthenocarpic fruit, undeveloped flowers and aborted flowers in tomato under moderately elevated temperatures. Sci. Hortic. 90:243-254. https://doi. org/10.1016/S0304-4238(00)00262-4

24. TEITEL, M.; LIRAN, O.; TANNY, J.; BARAK, M. 2008. Wind driven ventilation of a mono-span greenhouse with a rose crop and continuous screened side vents and its effect on flow patterns and microclimate. Biosystems Engineering. 101(1):111-122. https://doi. org/10.1016/j.biosystemseng.2008.05.012

25. TOMINAGA, Y.; MOCHIDA, A.; YOSHIE, R.; KATAOKA, H.; NOZU, T.; YOSHIKAWA, M.; SHIRASAWA, T. 2008. AIJ guidelines for practical applications of CFD to pedestrian wind environment around build- ings. J. Wind Engineering and Industrial Aerodynamics. 96(10-11):1749-1761. https://doi.org/10.1016/j. jweia.2008.02.058

26. VAN HENTEN, E.J.; BAKKER, J.C.; MARCELIS, L.F.M.; VAN TOOSTER, A.; DEKKER, E.; STANGHELLINI, C.; WESTRA, J. 2006. The adaptive greenhouse - an integrated systems approach to developing protected cultivation systems. Acta Horticulturae. 718:399-406. https://doi.org/ 10.17660/ActaHortic.2006.718.46.

27. VILLAGRÁN, E.A.; GIL, R.; ACUÑA, J.F.; BOJACÁ, C.R. 2012. Optimization of ventilation and its effect on the microclimate of a colombian multispan greenhouse. Agronomia Colombiana. 30(2):282-288.

Recibido: Mayo 7 de 2018

Aceptado: Octubre 23 de 2018

\section{Cómo citar:}

Villagran Munar, E.A.; Bojacá Aldana, C.R.; Rojas Bahamon, N.A. 2018. Determinacion del comportamiento térmico de un invernadero espacial colombiano mediante dinamica de fluidos computacional. Rev. U.D.C.A Act. \& Div. Cient. 21(2):415426. https://doi.org/10.31910/rudca.v21.n2.2018.1070 